\title{
Thermodynamics of two-colour QCD *
}

\author{
O. Kaczmarek with F. Karsch and E. Laermann \\ Fakultät für Physik, Universität Bielefeld, 33501 Bielefeld, Germany
}

\begin{abstract}
We discuss the thermodynamics of two-colour QCD with four flavours of staggered quarks on $8^{3} \times 4$ and $16^{3} \times 4$ lattices. In our simulations we use the Naik action for the fermions and a $(1,2)$ tree-level improved gauge action. We analyze the deconfinement and chiral phase transitions for four different quark masses $(\mathrm{m}=0.1,0.05,0.025,0.015)$. Contrary to three-colour QCD the peak in the Polyakov loop susceptibility decreases with decreasing quark mass. This reflects an early breaking of the string in the heavy quark potential, which we verify explicitly by calculating the heavy quark potential at finite temperature using Polyakov loop correlations.
\end{abstract}

\section{Introduction}

We investigate the thermodynamics of QCD with four flavours and colour group $S U(2)$ in the staggered discretisation [1]. In the continuum limit the phase transition is expected to be of first order induced by fluctuations [2].

In our simulations we used the exact hybridMC algorithm with a Symanzik improved $(1,2)$ gluon action and the Naik improvement for the fermionic sector. To reduce the number of conjugate gradient steps we used the minimal residual extrapolation method (MRE)[3]. The residuum for the $\mathrm{CG}$ was $10^{-8}$ inside the molecular dynamic trajectory and $10^{-13}$ elsewhere. Each trajectory was of length 1 , while the number of steps in the molecular dynamic evolution was adjusted to get a good acceptance.

For the analysis of the phase transition lattices of size $8^{3} \times 4$ with three different bare quark masses $m=0.1,0.05,0.025$ and size $16^{3} \times 4$ with $m=0.015$ were used to calculate the Polyakov loop, the chiral condensate and their susceptibilities. We used a noisy estimator with 25 random sources to calculate the susceptibility of $\bar{\psi} \psi$. Simulations on $16^{3} \times 4$ lattices at $m=0.025$ were performed to calculate finite temperature potentials from Polyakov loop correlations. For comparison of these potentials we also used results of two flavour QCD with colour group $S U(3)$ and standard gluonic and fermionic actions [4].

\footnotetext{
*This work was partly supported by the Deutsche Forschungsgemeinschaft under grant Ka 1198/3-1 and the EU TMR network grant ERBFMRX-CT97-0122.
}

\section{Polyakov Loop}

The results for the Polyakov loop are shown in Fig.1. With decreasing mass they seem to get flatter showing no clear sign of a phase-transition. For small $\beta$-values the Polyakov loop does not drop to zero which is a first hint for string breaking. Comparing the data for the susceptibility of

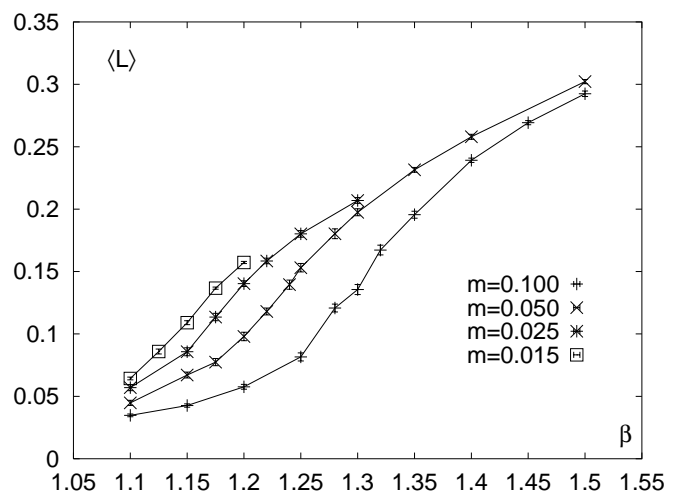

Figure 1. Polyakov loop on $8^{3} \times 4$ lattices $(\mathrm{m}=0.1$, $0.05,0.025)$ and on $16^{3} \times 4$ lattices $(\mathrm{m}=0.015)$.

the Polyakov loop for $S U(2)$ with four flavours (Fig.2) and $S U(3)$ with two flavours [5] one sees quite a different mass dependence of the peak heights. For $S U(2)$ the peaks decrease with decreasing mass while for $S U(3)$ the heights have the tendency to increase slightly. This reflects a smooth crossover from the low to the high temperature phase and an early breaking of the string for $S U(2)$ (see Fig.5). Further simulations are 


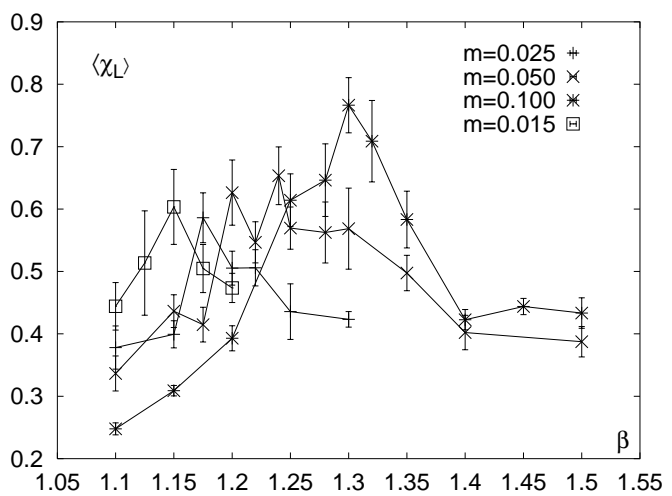

Figure 2. Susceptibility of the Polyakov loop on $8^{3} \times 4$ lattices $(\mathrm{m}=0.1,0.05,0.025)$ and on $16^{3} \times 4$ lattices $(\mathrm{m}=0.015)$.

needed to see if this behaviour is also valid for larger $N_{\tau}$ or if it could be a strong coupling effect.

\section{Chiral Condensate}

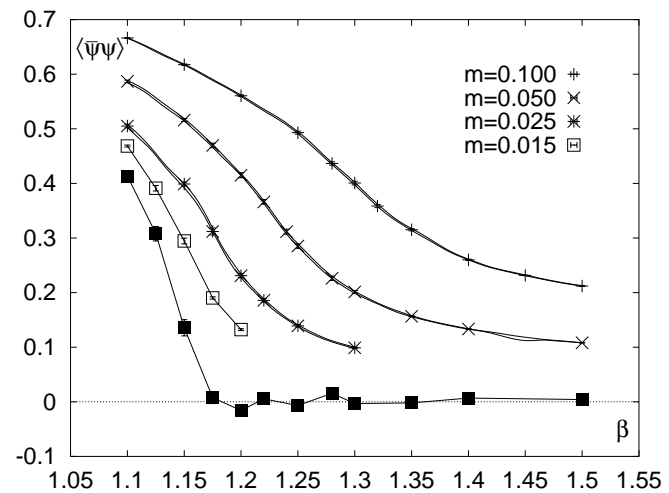

Figure 3. Chiral condensate for $\mathrm{m}=0.1,0.05$, $0.025\left(8^{3} \times 4\right), \mathrm{m}=0.015\left(16^{3} \times 4\right)$ and the linear extrapolation to $\mathrm{m}=0$.

The data for the chiral condensate in Fig.3 show a clear phase transition in the chiral limit although $\langle\bar{\psi} \psi\rangle$ stays finite for finite bare quark masses. The results for the two smallest masses were used to extrapolate to the zero mass limit with a linear ansatz

$\langle\bar{\psi} \psi\rangle(m=0)=\langle\bar{\psi} \psi\rangle(m)-c \cdot m$.

For $\beta>1.2$ chiral symmetry is restored in the zero mass limit within errors. A wider range of masses are needed to use other ansätze for the extrapolation.

So far, our results are compatible with earlier investigations by Kogut et al. [6]. In contrast to

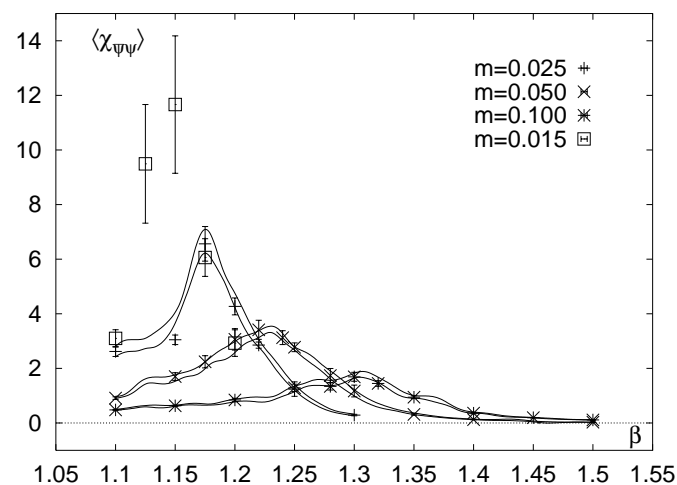

Figure 4. Susceptibility of the chiral condensate for $\mathrm{m}=0.1,0.05,0.025\left(8^{3} \times 4\right), \mathrm{m}=0.015\left(16^{3} \times 4\right)$.

the susceptibility of $L$ the comparison of $S U(2)$ and $S U(3)$ data 5 shows no major difference for the susceptibility of the chiral condensate. Both exhibit clear peaks that increase with decreasing bare quark mass. Also quantitatively the quark mass dependence of the peak heights is quite similar for both gauge groups. We have fitted the peak heights to the ansatz

$\chi_{\bar{\psi} \psi, \max }(m) \sim m^{-z_{m}}$.

\begin{tabular}{|c|c|c|}
\hline Action & Lattice & $z_{m}$ \\
\hline$S U(2), N_{f}=4$ & $8^{3} \times 4$ & $0.95(7)$ \\
$S U(3), N_{f}=2$ & $8^{3} \times 4$ & $0.84(5)$ \\
$S U(3), N_{f}=2$ & $16^{3} \times 4$ & $0.93(8)$ \\
\hline
\end{tabular}

Table 1. Fit-results of ansatz (2).

The results of the fits for $S U(2)$ and $S U(3)$ are shown in Tab.1. Although we do not expect a second order transition for 4-flavour, $N_{c}=2$ QCD in the continuum limit, we find no indication for a discontinuity, i.e. a first order phase transition. This may indicate that the transition is still controlled by the $U(1)$ symmetry of the staggered fermion action, i.e. we are still at too 
strong coupling.

\section{Finite Temperature Potentials}

We have calculated finite temperature potentials on $16^{3} \times 4$ lattices for $m=0.025$ and $T<T_{c}$ using Polyakov loop correlations up to a distance of $R T=2.0$

$P L C(R)=\left\langle L^{\dagger}(0,0,0) L\left(d x_{1}, d x_{2}, d x_{3}\right)\right\rangle$

with $d x_{i}=0 . .4$ and on-axis $d x_{i}=0 . .8$.

The potentials are extracted from the following relation:

$V(R)=-\frac{1}{N_{\tau}} \log \langle P L C(R)\rangle$.

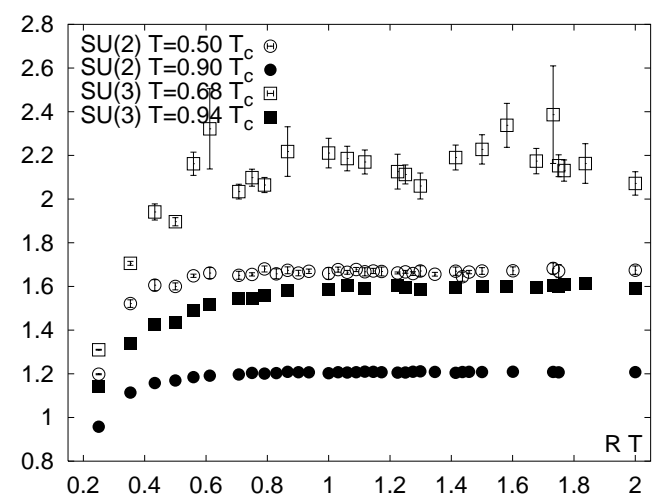

Figure 5. Comparison of the Potentials $V(R)$ for $N_{f}=4-S U(2)$ and $N_{f}=2-S U(3)$ for $T \approx T_{c}$ and smaller temperatures.

We compare our potentials with results for two flavour QCD 4. For both theories we see clear signs of string breaking. The potentials seem to rise up to a critical distance $R_{c}$ where the string breaks and $V\left(R>R_{c}\right)$ stays constant. It seems that for $S U(2)$ the string breaks at smaller distances than for $S U(3)$ which may be attributed to the larger number of light states in the $S U(2)$ theory that can contribute to the string breaking. It is however not yet clear how the heights of the potentials are controlled by self-energy contributions.

\section{Conclusions}

So far we have no indications for a first order phase transition for QCD with four flavours and colour group $S U(2)$. This may be a strong coupling artifact due to the incomplete chiral symmetry of the staggered action. Further investigations with larger temporal extent are needed to get closer to the continuum limit.

A rather striking feature of the $S U(2)$ theory is the decoupling of chiral symmetry restoring and deconfining aspects of the transition. While the chiral order parameter changes more rapidly close to $T_{c}$ with decreasing quark mass the Polyakov loop expectation values become flatter in this region.

Our data are compatible with earlier results by Kogut et al. [6]. Simulations at smaller quark masses should be performed to see if a first order phase transition appears as suggested by Kogut [7].

The finite temperature potentials show signs of string breaking for $S U(2)$ in agreement with the $S U(3)$ data of C. DeTar et al. [4]. Due to the larger number of light states in $S U(2)$ it seems that the string breaks earlier and the plateaus are lower than for $S U(3)$.

So far this is a qualitative comparison. One has to analyze the self-energy contributions to the potentials and needs information about the lattice spacing to really compare both.

\section{REFERENCES}

1. U. Heller, F. Karsch, Nucl.Phys.B285 (1985) 29.

2. R.D. Pisarski, F. Wilczek, Phys.Rev.D.29 (1984) 338 .

3. R.C. Brower, T. Ivanenko, A.R. Levi, K.N. Orginos, Nucl.Phys.B484 (1997) 353.

4. C. DeTar, O. Kaczmarek, F. Karsch, E. Laermann, hep-lat 9808028.

5. F. Karsch, E. Laermann, Phys.Rev.D50 (1994) 6954.

6. J. Kogut, J. Polonyi, H.W. Wyld, D.K. Sinclair, Nucl.Phys.B265(1986)293.

7. J. Kogut, Nucl.Phys.B290 (1987) 1. 